\title{
Single-Access Transumbilical Diagnostic Laparoscopy for Pancreatic Tumor Using Curved and Reusable Instruments
}

\author{
Giovanni Dapri, MD, FACS ${ }^{1}$, Pietro Carnevali, BSc ${ }^{1,2}$, Jacques Himpens, MD ${ }^{1}$, Paolo Bianchi, MD ${ }^{3}$, \\ Enrico Opocher, $\mathrm{MD}^{2}$, Marco Montorsi, $\mathrm{MD}^{4}$, and Guy-Bernard Cadière, $\mathrm{MD}$, $\mathbf{P h D}^{1}$
}

${ }^{1}$ Department of Gastrointestinal Surgery, European School of Laparoscopic Surgery, Saint-Pierre University Hospital, Brussels, Belgium; ${ }^{2}$ Department of Surgery, San Paolo University Hospital, Milan, Italy; ${ }^{3}$ Minimally Invasive Surgery Unit, European Institute of Oncology, Milan, Italy; ${ }^{4}$ Department of Surgery, Humanitas University Institut, Rozzano, Italy

\begin{abstract}
Introduction. Diagnostic laparoscopy in pancreatic tumors remains controversial. The main argument in favor of this procedure is that it helps prevent a delay of chemotherapy in cases of unresectable tumors or peritoneal/lymph node metastasis. We report a technique of performing this exploration through single-incision laparoscopy.

Video. The umbilicus is incised, and a purse-string suture is applied. An 11-mm nondisposable trocar is inserted for a $10-\mathrm{mm}, 30^{\circ}$ angled scope. Curved and reusable instruments (Karl Storz-Endoskope, Tuttlingen, Germany) are inserted transumbilically. Laparoscopic exploration of the cavity allows the visualization of suspected peritoneal or lymph node metastasis. Peritoneal lavage for cytology is performed. Biopsy is accomplished through the curved shape of the instruments, which establishes the working triangulation inside the abdomen as well as externally.
\end{abstract}

Laparoscopic ultrasonography of the liver and of the pancreas (after opening the lesser sac) is performed after replacement of the 11-mm trocar with a 13-mm trocar and the use of a 5-mm scope. The procedure can be continued either by laparoscopy or by open surgery. At completion, the umbilicus is meticulously closed to avoid complications.

Results. Operative time is $45-60$ minutes, blood loss is minimal, and the size of the umbilical incision is less than $15 \mathrm{~mm}$.

Conclusions. In case of unresectable tumors or peritoneal metastasis, single-access diagnostic laparoscopy for pancreatic tumors permits the start of chemotherapy after less than 7 days. Curved and reusable instruments allow the achievement of ergonomic conditions as classic laparoscopy, without increasing of conventional laparoscopic cost.

Electronic supplementary material The online version of this article (doi:10.1245/s10434-010-1344-1) contains supplementary material, which is available to authorized users.

(C) Society of Surgical Oncology 2010

First Received: 23 April 2010;

Published Online: 16 November 2010

G. Dapri, MD, FACS

e-mail: giovanni@dapri.net 